\title{
REAL PLACES AND TORUS BUNDLES
}

\author{
DANNY CALEGARI
}

\begin{abstract}
If $M$ is a hyperbolic once-punctured torus bundle over $S^{1}$, then the trace field of $M$ has no real places.
\end{abstract}

\section{INTRODUCTION}

This paper studies a particular example of the interaction between topology and number theory in the context of finite volume hyperbolic 3-manifolds. If a noncompact hyperbolic 3-manifold $M$ is irreducible and atoroidal, and homeomorphic to the interior of a compact 3-manifold with torus boundary components, then $M$ admits a unique complete hyperbolic structure. This structure determines a faithful representation from $\pi_{1}(M)$ into $\operatorname{PSL}(2, \mathbb{C})$ which can be taken to have image in $\operatorname{PSL}(2, K)$ for some smallest number field $K$, called the trace field of $M$. See [13], Theorem 4.2.3. (Note that the noncompactness of $M$ is important for the identification of $K$ with the trace field of $\pi_{1}(M)$.)

It is an important question to study which number fields $K$ can arise from this construction, and conversely, to understand the relationship between the topology of $M$ and the algebra of $K$. This question seems to be wide open, and very few nontrivial relationships are known.

In this paper we show by an elementary geometric argument that when $M$ is a hyperbolic once-punctured torus bundle over $S^{1}$, the trace field $K$ has no real places (i.e. it is not Galois conjugate to a subfield of $\mathbb{R}$ ).

1.1. Statement of results. In $\S 2$ we give an exposition of the relation between Euler classes, Stiefel-Whitney classes, orientations, and boundary traces for hyperbolic surfaces and 3-manifolds. All this material is classical, but it seems that there is no explicit and thorough account of it in the literature which takes our particular viewpoint. This discussion takes up more than half the length of the paperwe feel that its inclusion is justified by its potential interest as a reference for the 3-manifold community.

Note that some facts which might be otherwise somewhat obscure, are clarified by a thorough discussion of this foundational material. In particular, it is immediate from our viewpoint that for a hyperbolic knot complement $M$, the trace of a longitude is -2 , after lifting a geometric representation $\pi_{1}(M) \rightarrow \operatorname{PSL}(2, \mathbb{C})$ to $\mathrm{SL}(2, \mathbb{C})$.

In $\S 3$ we specialize to once-punctured torus bundles. After a preliminary discussion of trace fields and invariant trace fields, we prove our main theorem:

Date: 2/16/2006, Version 0.15 . 
Theorem A. Let $M_{\phi}$ be a hyperbolic oriented once-punctured torus bundle over $S^{1}$ with monodromy $\phi$. Let $K$ denote the trace field of $M_{\phi}$, and $k$ the invariant trace field. Then:

(1) K admits no real places

(2) If $k$ admits a real place, then $H_{1}(M)$ contains 2-torsion

In $\S 4$ we make some related observations. Most interesting is the observation that if $M$ contains a pseudo-rational surface - i.e. a surface $S$ with all traces rational - then such a surface has maximal Euler class with respect to any $\operatorname{PSL}(2, \mathbb{R})$ representation. In particular, a 3-manifold containing a pseudo-rational surface which is not Thurston norm minimizing in its homology class (e.g. it might be separating) has a trace field with no real place. A nice corollary, pointed out by A. Reid, is that this equality lets us construct examples of knot complements which contain no totally geodesic immersed surfaces, for example, the knot $8_{20}$ in Rolfsen's tables.

We also give an example showing that for every $n<0$ and every $m$ with $|m| \leq n$ and $m=n \bmod 2$ there are incompressible surfaces $S_{n, m}$ with Euler characteristic $n$ in some hyperbolic 3-manifold $M$ with a real place $\sigma$ for which $e_{\sigma}\left(S_{n, m}\right)=m$. The Milnor-Wood inequality implies that $|m| \leq n$ is sharp, and the mod 2 condition is implied by the fact that the representations are Galois conjugate into a geometric representation, so these are all the possibilities which can arise. Somewhat amazingly, all these examples can be found in a single manifold $M$, the complement of the link $8_{6}^{2}$ in Rolfsen's tables.

1.2. Acknowledgements. Thanks to J. Button, N. Dunfield, O. Goodman, A. Reid and the anonymous referee for comments, computations and corrections. Extra special thanks to A. Reid for nursing this paper through an problematic gestation, and for the idea of Corollary 4.6.

\section{EULER, STIEFEL-WHitNey, TRACES}

In what follows, we frequently deal with complete noncompact hyperbolic surfaces $S$ with finite area. Such surfaces are homeomorphic to the interior of a com-

pact surface $\bar{S}$ with boundary. By abuse of notation, we will refer to the boundary components of the (compactifying) surface $\bar{S}$ as the boundary components of $S$.

For the convenience of the reader, we give a thorough exposition of the relationship between SL, PSL, Stiefel-Whitney, Euler, and hyperbolic geometry. Related references are [8], [5].

2.1. Geometric representation. Let $S$ be a noncompact orientable surface of finite type, and let $\phi: S \rightarrow S$ be a pseudo-Anosov homeomorphism. Then the mapping torus

$$
M_{\phi}:=S \times I /(s, 1) \sim(\phi(s), 0)
$$

admits a complete hyperbolic structure. Corresponding to this hyperbolic structure there is a discrete faithful representation

$$
\rho_{\text {geo }}: \pi_{1}\left(M_{\phi}\right) \rightarrow \operatorname{PSL}(2, \mathbb{C})
$$

which is unique up to conjugacy and orientation. We fix one such representation, and call it the geometric representation. 
2.2. Quasifuchsian representations. An orientable surface $S$ with negative Euler characteristic itself admits a hyperbolic structure, and any such hyperbolic structure determines some discrete faithful representation

$$
\rho_{S}: \pi_{1}(S) \rightarrow \operatorname{PSL}(2, \mathbb{R})
$$

for which the image of each boundary curve is parabolic.

The space of discrete faithful representations from $\pi_{1}(S)$ into $\operatorname{PSL}(2, \mathbb{C})$ up to conjugacy and orientation, and for which the image of each boundary curve maps to a parabolic element, is connected, and contains an open dense subset homeomorphic to a ball consisting of quasifuchsian representations. For each quasifuchsian representation $\rho_{q}: \pi_{1}(S) \rightarrow \operatorname{PSL}(2, \mathbb{C})$ the ideal circle of the universal cover $\widetilde{S}$ maps to a quasicircle in the sphere $\mathbb{C P}^{1}$. See [1] and [14] for background and details on the theory of Kleinian groups.

2.3. Nonorientable representations. A surface $S$ might be orientable and yet admit representations into Isom $\left(\mathbb{H}^{2}\right)$ with nonorientable holonomy. The group of all isometries of $\mathbb{H}^{2}$ is $\operatorname{PGL}(2, \mathbb{R})$ which embeds into $\operatorname{PGL}(2, \mathbb{C}) \cong \operatorname{PSL}(2, \mathbb{C})$.

2.4. Classifying spaces. We may embed both $\operatorname{PSL}(2, \mathbb{R})$ and $\operatorname{PGL}(2, \mathbb{R})$ into the group Homeo $\left(S^{1}\right)$ by considering their action on the ideal circle $S_{\infty}^{1}$ of $\mathbb{H}^{2}$. The image of $\operatorname{PSL}(2, \mathbb{R})$ is contained in $\mathrm{Homeo}^{+}\left(S^{1}\right)$ (the superscript + denotes orientation preserving homeomorphisms). These inclusion maps are homotopy equivalences. The circle $S^{1}$ embeds in $S^{2}$ as the equator, and the group Homeo $\left(S^{1}\right)$ embeds in $\mathrm{Homeo}^{+}\left(S^{2}\right)$. We have two commutative diagrams

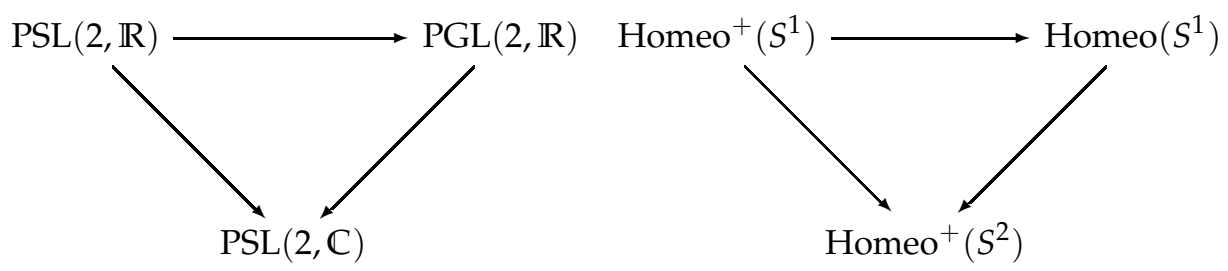

where the left diagram includes into the right diagram by a homotopy equivalence.

It is further true that the inclusions

$$
\mathrm{Homeo}^{+}\left(S^{1}\right) \rightarrow \mathrm{Homeo}^{+}\left(D^{2}\right)
$$

and

$$
\text { Homeo }^{+}\left(S^{2}\right) \rightarrow \text { Homeo }^{+}\left(D^{3}\right)
$$

where $D^{2}, D^{3}$ are open balls, obtained by coning to the center and throwing away the boundary, are homotopy equivalences. The first case is straightforward; the second follows from Hatcher's proof of the Smale conjecture [10] and the (homotopy) equivalence of the categories DIFF and TOP in dimension 3.

For any group $G$, and any space $X$, a representation

$$
\rho: \pi_{1}(X) \rightarrow G
$$

induces a homotopy class of maps to the classifying space BG. There is a tautological $G$ bundle over BG called EG which pulls back to a $G$-bundle over X called $E_{\rho}$. Topologically, we form $E_{\rho}$ as the quotient bundle

$$
E_{\rho}=\widetilde{X} \times G /(x, g) \sim(\alpha(x), \rho(\alpha)(g))
$$


where $\alpha$ ranges over elements of $\pi_{1}(X)$.

2.5. Homotopy of BHomeo. The group $\mathrm{Homeo}^{+}\left(S^{1}\right)$ is homotopy equivalent to the subgroup $\mathrm{SO}(2, \mathbb{R}) \approx S^{1}$ consisting of rotations. Homeo $\left(S^{1}\right)$ is homotopy equivalent to the subgroup $\mathrm{O}(2, \mathbb{R})$ which is homotopic to two disjoint circles. Homeo $^{+}\left(S^{2}\right)$ is homotopy equivalent to $S \mathrm{O}(3, \mathbb{R}) \approx \mathbb{R P}^{3}$. It follows that we can compute the homotopy groups of BHomeo:

$$
\begin{gathered}
\pi_{i}\left(\text { BHomeo }^{+}\left(S^{1}\right)\right)=\left\{\begin{array}{ll}
\mathbb{Z} & \text { if } i=2 \\
0 & \text { otherwise }
\end{array} \quad \pi_{i}\left(\operatorname{BHomeo}\left(S^{1}\right)\right)= \begin{cases}\mathbb{Z} / 2 \mathbb{Z} & \text { if } i=1 \\
\mathbb{Z} & \text { if } i=2 \\
0 & \text { otherwise }\end{cases} \right. \\
\pi_{i}\left(\text { BHomeo }^{+}\left(S^{2}\right)\right)= \begin{cases}\mathbb{Z} / 2 \mathbb{Z} & \text { if } i=2 \\
0 & \text { if } i=0,1,3 \\
\mathbb{Z} & \text { if } i=4\end{cases}
\end{gathered}
$$

and is torsion for $i>4$.

Cohomology on BG pulls back to cohomology classes on $X$ which represent the first obstruction to trivializing the bundle $E_{\rho}$. Since $\mathrm{BHomeo}^{+}\left(S^{1}\right)$ is a $K(\mathbb{Z}, 2)$ and therefore has the homotopy type of $\mathbb{C P}^{\infty}$, the cohomology ring is generated by a single element $e \in H^{2}\left(\mathrm{BHomeo}^{+}\left(S^{1}\right) ; \mathbb{Z}\right)$. Identifying $\mathrm{Homeo}^{+}\left(S^{1}\right)$ with $\operatorname{PSL}(2, \mathbb{R})$ up to homotopy, this element represents the obstruction to lifting a representation from $\operatorname{PSL}(2, \mathbb{R})$ to $\widetilde{\mathrm{SL}}(2, \mathbb{R})$, its universal covering group. The mod 2 reduction of $e$ is the obstruction to lifting to $\operatorname{SL}(2, \mathbb{R})$.

Since $\mathrm{BHomeo}^{+}\left(S^{2}\right)$ is a $K(\mathbb{Z} / 2 \mathbb{Z}, 2)$ below dimension 4 , only the class $w \in$ $H^{2}\left(\right.$ BHomeo $\left.^{+}\left(S^{2}\right) ; \mathbb{Z} / 2 \mathbb{Z}\right)$ is relevant to 3-manifolds. Identifying $\mathrm{Homeo}^{+}\left(S^{2}\right)$ with $\operatorname{PSL}(2, \mathbb{C})$ up to homotopy, this represents the obstruction to lifting a representation from $\operatorname{PSL}(2, \mathbb{C})$ into $\operatorname{SL}(2, \mathbb{C})$. If we include $\operatorname{PSL}(2, \mathbb{R})$ into $\operatorname{PSL}(2, \mathbb{C})$ then we see that $w$ is the image of $e$ under $H^{2}(X ; \mathbb{Z}) \rightarrow H^{2}(X ; \mathbb{Z} / 2 \mathbb{Z})$.

2.6. BHomeo $\left(S^{1}\right)$. The short exact sequence

$$
0 \rightarrow \operatorname{Homeo}^{+}\left(S^{1}\right) \rightarrow \operatorname{Homeo}\left(S^{1}\right) \rightarrow \mathbb{Z} / 2 \mathbb{Z} \rightarrow 0
$$

gives rise to a fibration of spaces

$$
\text { BHomeo }^{+}\left(S^{1}\right) \rightarrow \operatorname{BHomeo}\left(S^{1}\right) \rightarrow \mathbb{R P}^{\infty}
$$

which exhibits $\mathrm{BHomeo}\left(S^{1}\right)$ up to homotopy as a twisted $\mathrm{CP}^{\infty}$ bundle over $\mathbb{R} \mathbb{P}^{\infty}$. The generator of $\pi_{1}\left(\mathrm{BHomeo}\left(S^{1}\right)\right)$ acts on $\pi_{2}\left(\mathrm{BHomeo}\left(S^{1}\right)\right)$ by multiplication by -1 . Since this multiplication is trivial with $\mathbb{Z} / 2 \mathbb{Z}$ coefficients, the $\mathbb{Z} / 2 \mathbb{Z}$ cohomology can be computed from the Künneth formula. In low dimensions we get

$$
H^{i}\left(\operatorname{BHomeo}\left(S^{1}\right) ; \mathbb{Z} / 2 \mathbb{Z}\right)= \begin{cases}\mathbb{Z} / 2 \mathbb{Z} & \text { for } i=0,1 \\ \mathbb{Z} / 2 \mathbb{Z} \oplus \mathbb{Z} / 2 \mathbb{Z} & \text { for } i=2\end{cases}
$$

The generator in dimension 1 is the orientation class $o$, and the generators in dimension 2 are $o^{2}$ and $r$ which is the mod 2 reduction of the Euler class, and is obtained by pulling back $w$ from $H^{2}\left(\mathrm{BHomeo}^{+}\left(S^{2}\right) ; \mathbb{Z} / 2 \mathbb{Z}\right)$. 
2.7. Relative bundles. Suppose we have a pair of spaces $X, Y$ and a representation

$$
\rho: \pi_{1}(X) \rightarrow G
$$

such that $\left.\rho\right|_{\pi_{1}(Y)}$ is trivial. Then the resulting map to the classifying space maps $Y$ to the basepoint of $B G$, and there is a well-defined relative homotopy class of pairs. This defines a canonical trivialization of the restricted bundle $\left.E_{\rho}\right|_{Y}$. It follows that we can pull back reduced cohomology of BG to a relative class in $H^{*}(X, Y)$ which represents the obstruction to extending this trivialization of $E_{\rho}$ over $Y$ to all of $X$.

More generally, suppose $G$ contains a closed contractible subgroup $H$. Then the coset space $G / H$ is homotopy equivalent to $G$, and the bundle $E_{\rho}$ can be replaced by a homotopy equivalent bundle $E_{\rho} / H$. A representation $\rho: \pi_{1}(X) \rightarrow G$ for which $\rho\left(\pi_{1}(Y)\right)$ is contained in $H$ defines a canonical trivialization of $E_{\rho} / H$, and therefore a relative cohomology class in $H^{*}(X, Y)$.

In the groups $\operatorname{PSL}(2, \mathbb{R})$ and $\operatorname{PSL}(2, \mathbb{C})$ the maximal parabolic subgroups are contractible; these are the $N$ subgroups with respect to the $K A N$ (or Iwasawa) decomposition of these groups.

In $\mathrm{Homeo}^{+}\left(S^{1}\right)$ the stabilizer of a point is contractible. In $\mathrm{Homeo}^{+}\left(S^{2}\right)$, the stabilizer of a point is not contractible, being homotopic to $S^{1}$.

2.8. Euler classes of surfaces, and relative Euler class. For $S$ a closed orientable hyperbolic surface, the Euler class $e_{\rho_{S}}$ of the representation

$$
\rho_{S}: \pi_{1}(S) \rightarrow \operatorname{PSL}(2, \mathbb{R})
$$

associated to any hyperbolic structure on $S$ satisfies

$$
e_{\rho_{S}}([S])= \pm \chi(S)
$$

where $[S]$ represents the fundamental class in $H_{2}(S ; \mathbb{Z})$, and the sign depends on the choice of orientation. Note in this case that $E_{\rho_{S}}$ is isomorphic to the unit tangent bundle of $S$.

If $S$ is orientable and complete with finite area, but possibly with punctures, then there is a relative fundamental class in $H_{2}(S, \partial S ; \mathbb{Z})$. If $E_{\rho_{S}}$ represents the associated circle bundle, then $E_{\rho_{S}}$ restricts to a finite union of tori fibering over the boundary components of $S$.

By our assumption, the holonomy around a boundary component is parabolic, and has a (unique) fixed point in $S^{1}$. This fixed point suspends to a canonical section of $E_{\rho_{S}}$ over $\partial S$, and defines a trivialization of this restricted bundle. The relative Euler class of $\rho_{S}$ is the element of $H^{2}(S, \partial S ; \mathbb{Z})$ which represents the obstruction to extending this trivialization of $\left.E_{\rho_{S}}\right|_{\partial S}$ over all of $E_{\rho_{S}}$.

2.9. Geometric computation of Euler class. In [19], Thurston defined a 2-cocycle for a group $G$ acting in an orientation-preserving way on $S^{1}$. If $\sigma: G \rightarrow \operatorname{Homeo}^{+}\left(S^{1}\right)$ is a representation, choose a point $p \in S^{1}$ and for every triple $g_{0}, g_{1}, g_{2} \in G$ define

$$
c\left(g_{0}, g_{1}, g_{2}\right)= \begin{cases}1 & \text { if } g_{0}(p), g_{1}(p), g_{2}(p) \text { are positively ordered } \\ -1 & \text { if } g_{0}(p), g_{1}(p), g_{2}(p) \text { are negatively ordered } \\ 0 & \text { if } g_{0}(p), g_{1}(p), g_{2}(p) \text { are degenerate }\end{cases}
$$

Equivalently, think of $S^{1}$ as the ideal boundary of $\mathbb{H}^{2}$. Then for $g_{0}, g_{1}, g_{2}$ a triple of elements in $G$, define $c\left(g_{0}, g_{1}, g_{2}\right)$ to be the (signed) area of the ideal triangle with vertices at the $g_{i}(p)$, divided by $\pi$. 
From this geometric definition, it is easy to see that $c$ is coclosed, and that the cohomology class it defines is independent of $p$. Suppose that $G=\pi_{1}(M)$ and $H_{i}<G$ is $\pi_{1}\left(\partial M_{i}\right)$ for each component $\partial M_{i}$ of $\partial M$. If each $H_{i}$ has a fixed point in $S^{1}$, then for some choice of basepoint, $c$ is identically zero on $H_{i}$. It follows that $c$ defines a relative class in $H^{2}(M, \partial M ; \mathbb{Z})$.

Lemma 2.1. The Thurston cocycle $c$ is related to the Euler class e by

$$
[c]=2[e] \text { in } H^{2}(M, \partial M ; \mathbb{Z})
$$

For a proof, see e.g. [11]. For more details, and related constructions, see [7] or [3].

2.10. Stiefel-Whitney class and $\operatorname{SL}(2, \mathbb{C})$. For a closed hyperbolic 3-manifold, the $S^{2}$ bundle coming from the geometric representation $\rho_{\text {geo }}$ is isomorphic to the unit tangent bundle (to see this, use the exponential map). The pullback of the generator of $\mathrm{H}^{2}\left(\mathrm{BHomeo}^{+}\left(S^{2}\right) ; \mathbb{Z} / 2 \mathbb{Z}\right)$ can therefore be identified with the second Stiefel-Whitney class of $M$. If $M$ is orientable, $T M$ is parallelizable, so this class must vanish.

If $M$ is noncompact with finite volume, the geometric representation on each boundary torus group is parabolic, with a fixed point $p \in \mathbb{C P}^{1}$. The subgroup $N_{p}<\operatorname{PSL}(2, \mathbb{C})$ of parabolic elements fixing $p$ is contractible, so there is a trivialization of the associated bundle over $\partial M$ and we get a relative second StiefelWhitney class

$$
w_{\text {geo }} \in H^{2}(M, \partial M ; \mathbb{Z} / 2 \mathbb{Z})
$$

which might not be trivial.

Note that the image of $w_{\text {geo }}$ in $H^{2}(M ; \mathbb{Z} / 2 \mathbb{Z})$ is the ordinary second StiefelWhitney class which is always trivial for orientable $M$, as above. It follows from the long exact sequence in cohomology that $w_{\text {geo }}$ is the image of a distinguished class in $H^{1}(\partial M ; \mathbb{Z} / 2 \mathbb{Z}) / i^{*} H^{1}(M ; \mathbb{Z} / 2 \mathbb{Z})$, where $i^{*}$ denotes the homomorphism induced by the inclusion map $i: \partial M \rightarrow M$.

Since the ordinary second Stiefel-Whitney class vanishes, the geometric representation lifts to the double cover

$$
\hat{\rho}_{\text {geo }}: \pi_{1}(M) \rightarrow \mathrm{SL}(2, \mathbb{C})
$$

Since every element of $\pi_{1}(\partial M)$ is parabolic, the preimages either have trace 2 or -2 . Different choices of lift to $\operatorname{SL}(2, \mathbb{C})$ might change the value on elements of $H_{1}(\partial M ; \mathbb{Z} / 2 \mathbb{Z})$ which are in the image of $H_{1}(M ; \mathbb{Z} / 2 \mathbb{Z})$ but do not change the values on elements which are homologically nontrivial in $\partial M$ but bound in $M$ (mod 2). In particular, if $\alpha \in \pi_{1}(\partial M)$ represents zero in $H_{1}(M ; \mathbb{Z} / 2 \mathbb{Z})$, then the trace is independent of the choice of lift. We deduce that if trace $\left(\hat{\rho}_{\text {geo }}(\alpha)\right)=-2$ where $\alpha=\partial S$ for some properly embedded $S \subset M$ then $w_{\text {geo }}$ is nontrivial.

In general, for any representation

$$
\rho: \pi_{1}(M) \rightarrow \operatorname{PSL}(2, \mathbb{C})
$$

which sends $\pi_{1}(\partial M)$ to parabolic elements, we get a relative class

$$
w_{\rho} \in H^{2}(M, \partial M ; \mathbb{Z} / 2 \mathbb{Z})
$$

If $\rho$ lifts to $\hat{\rho}: \pi_{1}(M) \rightarrow \mathrm{SL}(2, \mathbb{C})$ then the image of $w_{\rho}$ in $H^{2}(M ; \mathbb{Z} / 2 \mathbb{Z})$ is zero. If trace $(\hat{\rho}(\alpha))=-2$ for some $\alpha \in \pi_{1}(\partial M)$ which represents a trivial class in $H_{1}(M ; \mathbb{Z} / 2 \mathbb{Z})$ then $w_{\rho}$ is nontrivial. We summarize this as a lemma: 
Lemma 2.2. Suppose that $M$ is a compact orientable 3-manifold with torus boundary components, and suppose $\rho$ is some representation

$$
\rho: \pi_{1}(M) \rightarrow \operatorname{PSL}(2, \mathbb{C})
$$

for which elements of $\pi_{1}(\partial M)$ map to parabolic transformations. Suppose further that $\rho$ lifts to

$$
\hat{\rho}: \pi_{1}(M) \rightarrow S L(2, \mathbb{C})
$$

Let $w_{\rho} \in H^{2}(M, \partial M ; \mathbb{Z} / 2 \mathbb{Z})$ denote the relative Stiefel-Whitney class of $\rho$, where the trivialization on $\partial M$ comes from the fixed point of the corresponding parabolic subgroup. Further, suppose there is some $\alpha \in \pi_{1}(\partial M)$ representing zero in $H_{1}(M ; \mathbb{Z} / 2 \mathbb{Z})$ for which

$$
\operatorname{trace}(\hat{\rho}(\alpha))=-2
$$

Then $w_{\rho}$ is nontrivial.

2.11. $\chi$ and $w$. Let $M$ be a manifold containing an incompressible surface $S$ with a single boundary component. It turns out that there is a very simple relationship between $\chi(S)$ and $w_{\text {geo }}([S])$; in fact, $w_{\text {geo }}([S])$ is just the mod 2 reduction of $\chi(S)$.

The simplest way to see this is topological. The value of $w_{\text {geo }}([S])$ depends only on the topology of the $S^{2}$ bundle over $S$ obtained from the representation

$$
\left.\rho_{\text {geo }}\right|_{S}: \pi_{1}(S) \rightarrow \operatorname{PSL}(2, \mathbb{C})<\operatorname{Homeo}^{+}\left(S^{2}\right)
$$

By using the exponential map, we may identify this bundle with the restriction of the unit tangent bundle $\left.U T M\right|_{S}$. In particular, the value of $w_{\text {geo }}([S])$ only depends on the topology of a tubular neighborhood of $S$ in $M$. If $S$ is two-sided and embedded, then this tubular neighborhood is just a product $S \times I$ and therefore the value of $w_{\text {geo }}([S])$ depends only on $S$. Now, for a geometric representation $\left.\rho_{S}\right|_{S}$ coming from a hyperbolic structure on $S$, we have already seen that $w_{S}([S])=\chi(S) \bmod 2$. In particular, we have the following lemma:

Lemma 2.3. Let $M$ be a complete finite-volume orientable hyperbolic 3-manifold. If $S \subset$ $M$ is incompressible and orientable, then

$$
w_{\text {geo }}([S])=\chi(S) \bmod 2
$$

where $[S]$ represents the fundamental class in $H_{2}(S, \partial S ; \mathbb{Z} / 2 \mathbb{Z})$.

The following corollary is folklore, and seems to have been observed first by $W$. Thurston, at least for Seifert surfaces of knots in $S^{3}$. There appears to be some confusion in the literature about whether it is well-known, and therefore we state it for completeness:

Corollary 2.4. Let $M$ be a complete noncompact orientable hyperbolic 3-manifold, and let $\alpha \in \partial M$ be the boundary of a 2-sided incompressible surface $S \subset M$. If $\hat{\rho}_{\text {geo }}: \pi_{1}(M) \rightarrow$ $S L(2, \mathbb{C})$ is any lift of the geometric representation, then

$$
\operatorname{trace}\left(\hat{\rho}_{g e o}(\alpha)\right)=-2
$$

It follows that the longitude of any knot has trace -2 . This answers Question 6.2 in [4].

We remark that by the proof of the Ending Lamination Conjecture [1], for $S$ incompressible, the geometric representation $\left.\rho_{\text {geo }}\right|_{S}$ coming from the hyperbolic structure on $M$ and a geometric representation $\left.\rho_{S}\right|_{S}$ coming from a hyperbolic 
structure on $S$ are homotopic through representations of $\pi_{1}(S)$ into $\operatorname{PSL}(2, \mathbb{C})$ which send boundary curves to parabolic elements, since $\left.\rho_{\text {geo }}\right|_{S}$ is always in the closure of the space of quasifuchsian representations. This gives another, more highpowered proof of Lemma 2.3.

\section{TORUS BUNDLES}

3.1. Number fields. If $M$ is a complete hyperbolic 3-manifold of finite volume, we have the corresponding geometric representation

$$
\rho_{\text {geo }}: \pi_{1}(M) \rightarrow \operatorname{PSL}(2, \mathbb{C})
$$

The trace field $K$ of $M$ is the field generated by the traces of $\rho_{\text {geo }}(\alpha)$, as $\alpha$ varies over the elements of $\pi_{1}(M)$. Of course, the trace of an element in $\operatorname{PSL}(2, \mathbb{C})$ is only determined up to sign, but the field generated by these elements is independent of sign.

It turns out that the trace field $K$ is always a number field (i.e. some finite algebraic extension of $\mathbb{Q}$ ) and as remarked in the introduction, for $M$ noncompact, $\rho_{\text {geo }}$ can be conjugated into $\operatorname{PSL}(2, K)$.

The invariant trace field is the subfield $k$ of $K$ generated by the squares of the traces of $\rho_{\text {geo }}(\alpha)$ as above. In general, $k$ and $K$ are not equal, and the degree satisfies $[K: k]=2^{n}$ for some $n$. The field $k$ is an invariant of the commensurability class of $M$, where two hyperbolic manifolds $M, N$ are said to be commensurable if they have a common finite cover.

See [13] for details and proofs.

3.2. Action of $\operatorname{Gal}(L / \mathbb{Q})$. Let $L$ denote the Galois closure of $K$ in $\mathbb{C}$. If $p(x)$ is the minimal polynomial of a generating element of $K$, then $L$ is obtained from $Q$ by adjoining all roots of $p(x)$.

The Galois group $\operatorname{Gal}(L / \mathbb{Q})$ of $L$ over $\mathbb{Q}$ acts on $L$ by field automorphisms, conjugating $K$ into different subfields of $\mathbb{C}$.

The various (Galois conjugate) embeddings of $K$ into $\mathbb{C}$ are called places, and can be real or complex. Complex places come in pairs, interchanged by complex conjugation. If the degree $[K: \mathbb{Q}]=d$, then

$$
d=r_{1}+2 r_{2}
$$

where $r_{1}$ is the number of real places, and $r_{2}$ is the number of conjugate pairs of complex places.

In general, if

$$
\rho: \pi_{1}(M) \rightarrow \operatorname{PSL}(2, K)
$$

is some representation which is parabolic on $\partial M$, and if the relative Stiefel-Whitney class $w_{\rho}$ has zero image in $H^{2}(M ; \mathbb{Z} / 2 \mathbb{Z})$, then $\rho$ lifts to $\hat{\rho}$, and parabolic elements lift to elements of $\operatorname{SL}(2, K)$ with trace equal to \pm 2 . If $\rho_{\sigma}$ is obtained from $\rho$ by Galois conjugating $K$ to $K_{\sigma}$, then $\rho_{\sigma}$ lifts to $\operatorname{SL}\left(2, K_{\sigma}\right)$, so the relative Stiefel-Whitney class $w_{\rho_{\sigma}}$ has zero image in $H^{2}(M ; \mathbb{Z} / 2 \mathbb{Z})$. Moreover, since \pm 2 are in the fixed field of $\sigma$, we have equality

$$
\hat{\rho}_{\sigma}(\alpha)=\hat{\rho}(\alpha)
$$

Since the relative class is determined by these traces, we have equality

$$
w_{\rho}=w_{\rho_{\sigma}}
$$


Remark 3.1. The invariance of $w_{\rho}$ under the Galois group implies that $w_{\rho}$ can be pulled back from the cohomology of $\operatorname{BPSL}(2, \overline{\mathrm{Q}})$ where $\overline{\mathrm{Q}}$ has the discrete topology.

3.3. Real places for $K$ and $k$. A real place for $K$ determines an embedding of $\pi_{1}(M)$ in $\operatorname{PSL}(2, \mathbb{R})$, which lifts to $\operatorname{SL}(2, \mathbb{R})$, by the vanishing of the second StiefelWhitney class for a 3-manifold, and the invariance of $w$ under the action of the $\operatorname{group} \operatorname{Gal}(L / \mathbb{Q})$.

Let $\Gamma$ be the group generated by squares of elements of $\pi_{1}(M)$ where $M$ is noncompact as before. A real place for $k$ determines an embedding of $\Gamma$ into $\operatorname{PSL}(2, \mathbb{R})$ which extends to an embedding of $\pi_{1}(M)$ into $\operatorname{PSL}(2, \mathbb{C})$ for which every element of $\pi_{1}(M)$ has a trace which is real or pure imaginary.

Since the square of every element $\alpha$ of $\pi_{1}(M)$ stabilizes $\mathbb{H}^{2}$ in $\mathbb{H}^{3}$, it follows that $\alpha$ either fixes $\mathbb{H}^{2}$ (possibly reversing orientation) or takes it to an orthogonal copy of $\mathbb{H}^{2}$. In the second case, the square $\alpha^{2}$ takes $\mathbb{H}^{2}$ to itself by an orientationreversing isometry, contrary to the fact that $\alpha^{2}$ is in $\operatorname{PSL}(2, \mathbb{R})$ by hypothesis. It follows that $\pi_{1}(M)$ preserves $\mathbb{H}^{2}$, and therefore has image in $\operatorname{PGL}(2, \mathbb{R})$.

Again, this discussion depends on the noncompactness of $M$. In general, for noncompact $M$, a similar argument shows that the representation of $\pi_{1}(M)$ can always be conjugated into $\operatorname{PGL}(2, k)$ where $k$ is the invariant trace field. This fact is implicit e.g. in [16], page 278.

3.4. Homology of torus bundles. Let $M_{\phi}$ be a hyperbolic once-punctured torus bundle with monodromy $\phi$. Then $\phi$ induces an automorphism on $H_{1}(T ; \mathbb{Z})$, represented by some matrix

$$
\phi \sim\left(\begin{array}{ll}
a & b \\
c & d
\end{array}\right)
$$

Since $\phi$ is pseudo-Anosov, the trace $a+d$ satisfies $|a+d|>2$. Then $H_{1}\left(M_{\phi}\right)$ is isomorphic to the kernel of the map

$$
\left(\begin{array}{ccc}
a-1 & b & 0 \\
c & d-1 & 0 \\
0 & 0 & 0
\end{array}\right): \mathbb{Z}^{3} \rightarrow \mathbb{Z}^{3}
$$

which has rank 1 , and therefore $H_{2}\left(M_{\phi}, \partial M_{\phi} ; \mathbb{Z}\right)$ is isomorphic to $\mathbb{Z}$, generated by the relative class of the fiber.

3.5. Torus bundles and 2-torsion. Let $M_{\phi}$ be a hyperbolic surface bundle over $S^{1}$ with fiber a once-punctured torus $T$. The following theorem relates topology, homological algebra, and number theory:

Theorem A. Let $M_{\phi}$ be a hyperbolic oriented once-punctured torus bundle over $S^{1}$ with monodromy $\phi$. Let $K$ denote the trace field of $M_{\phi}$, and $k$ the invariant trace field. Then:

(1) K admits no real places

(2) If $k$ admits a real place, then $H_{1}(M)$ contains 2-torsion

Proof. We suppose after conjugating that the image of $\rho_{\text {geo }}$ lies in $\operatorname{PSL}(2, K)$. We know that $\rho_{\text {geo }}$ lifts to

$$
\hat{\rho}_{\text {geo }}: \pi_{1}\left(M_{\phi}\right) \rightarrow \mathrm{SL}(2, K)
$$


Moreover, if $T$ denotes the fiber of the fibration, and $A, B$ are standard (free) generators for $\pi_{1}(T)$, then

$$
\text { trace } \hat{\rho}_{\text {geo }}([A, B])=-2
$$

as in Lemma 2.3.

Suppose $\sigma: K \rightarrow \mathbb{R}$ is a real place, and let

$$
\hat{\rho}_{\sigma}: \pi_{1}\left(M_{\phi}\right) \rightarrow \operatorname{SL}(2, \mathbb{R})
$$

be obtained by Galois conjugating $K$ into $\mathbb{R}$. Let $\rho_{\sigma}: \pi_{1}\left(M_{\phi}\right) \rightarrow \operatorname{PSL}(2, \mathbb{R})$ be obtained by composing $\hat{\rho}_{\sigma}$ with the covering map $\operatorname{SL}(2, \mathbb{R}) \rightarrow \operatorname{PSL}(2, \mathbb{R})$.

We denote the relative Euler class of $\rho_{\sigma}$ by $e$ :

$$
e \in H^{2}\left(M_{\phi}, \partial M_{\phi} ; \mathbb{Z}\right)
$$

Since $w_{\text {geo }}=w_{\sigma}$ we must have that $e([T])$ is odd.

Triangulate $T$ by two ideal triangles $\Delta_{1}, \Delta_{2}$. The representation $\rho_{\sigma}$ determines a developing map from the universal cover $\widetilde{T}$ to $\mathbb{H}^{2}$

$$
d: \widetilde{T} \rightarrow \mathbb{H}^{2}
$$

If the image of both triangles has the same orientation, then the developing map is a homeomorphism, and we obtain a complete hyperbolic structure on $T$ which is invariant under $\phi$. But this implies that $\phi$ has finite order, which is incompatible with the existence of a complete hyperbolic structure on $M_{\phi}$. It follows that the orientations on the images of the $\Delta_{i}$ disagree.

By Thurston's formula for $2 e$ (Lemma 2.1), we have

$$
2 e([T])=\sum_{i} \text { sign of orientation on } d\left(\Delta_{i}\right)=0
$$

This gives a contradiction, and shows that $K$ has no real place.

Now if $k$ admits a real place, then there is some $\sigma: K \rightarrow \mathbb{C}$ such that

$$
\rho_{\sigma}: \pi_{1}\left(M_{\phi}\right) \rightarrow \operatorname{PGL}(2, \mathbb{R})
$$

As above, we get a developing map from $\widetilde{T}$ to $\mathbb{H}^{2}$ for which the orientations on the ideal triangles must disagree, and the rational relative Euler class of the action must vanish. Since $\rho_{\sigma}$ is conjugate into $\operatorname{PGL}(2, \mathbb{R})$ but not $\operatorname{PSL}(2, \mathbb{R})$ the orientation class $o_{\sigma} \in H^{1}\left(M_{\phi} ; \mathbb{Z} / 2 \mathbb{Z}\right)$ must be nontrivial. In fact, since the traces of elements of $\pi_{1}\left(\partial M_{\phi}\right)$ are \pm 2 , boundary elements map to the $\operatorname{subgroup} \operatorname{PSL}(2, \mathbb{R})$, and therefore the orientation class $o_{\sigma}$ is a nontrivial class in $H^{1}\left(M_{\phi}, \partial M_{\phi} ; \mathbb{Z} / 2 \mathbb{Z}\right)$.

Since $H_{1}(M, \partial M ; \mathbb{Z})$ is torsion for a punctured torus bundle, we are done.

Remark 3.2. Note that any field of odd degree admits a real place; in particular, the degree of $K$ is always even.

Remark 3.3. If $M$ is a (compact or noncompact) hyperbolic surface bundle, and $S$ is any fiber, then the same argument shows that if $K$ has a real place $\sigma: K \rightarrow \mathbb{R}$, then

$$
\left|e_{\sigma}(S)\right|<-\chi(S), e_{\sigma}(S)=\chi(S) \bmod 2
$$

Remark 3.4. J. Button has studied trace fields of punctured torus bundles with monodromy of the form $L^{-1} R^{-n}$ in [2]. He showed for positive $n \equiv 2 \bmod 4$ and for all odd $n$ that the invariant trace field of $\pi_{1}\left(M_{n}\right)$ has no real places. For $n$ odd, the 
homology of $M_{n}$ has no 2-torsion, but for $n$ even and not divisible by 4 , this does not follow from Theorem A, but rather from an explicit computation.

One might further ask whether every punctured torus bundle whose invariant trace field has a real place has 4-torsion in $H_{1}$. In fact, we posed exactly this question in an earlier version of this paper. J. Button has found a counterexample to this question: the census manifold s299 is a once-punctured torus bundle with monodromy $-R^{4} L^{2}$ whose invariant trace field $k$ has degree 3 , and whose trace field $K$ has degree 12 , and which has first homology $\mathbb{Z} \oplus \mathbb{Z} / 2 \mathbb{Z} \oplus \mathbb{Z} / 6 \mathbb{Z}$.

Remark 3.5. In [8], W. Goldman characterizes geometric representations of once punctured torus groups amongst all $\operatorname{PSL}(2, \mathbb{R})$ representations in terms of trace data. This gives an alternate proof of the first part of Theorem A, without using the geometric formula for the Euler class.

Remark 3.6. Part (2) of Theorem A also follows from Corollary 2.3 of [16].

Example 3.7. Amongst the cusped manifolds in the Hodgson-Weeks census (see [20]), m039 is a torus bundle with monodromy $R L^{4}, H_{1}=\mathbb{Z} \oplus \mathbb{Z} / 4 \mathbb{Z}$ and invariant trace field with minimal polynomial $x^{3}-x^{2}+x+1$. It has a degree 2 cover v3225 for which this is the trace field; this cover is fibered with fiber a twice-punctured torus, so necessarily the Euler class of the representation associated to the real place must vanish on this fiber.

Some other examples: $\mathrm{m} 040$ is a torus bundle with monodromy $-R L^{4}, H_{1}=$ $\mathbb{Z} \oplus \mathbb{Z} / 8 \mathbb{Z}$ and invariant trace field with minimal polynomial $x^{3}-x^{2}+x+1$, and v2231 is a torus bundle with monodromy $R L^{2} R L^{3}, H_{1}=\mathbb{Z} \oplus \mathbb{Z} / 16 \mathbb{Z}$ and invariant trace field with minimal polynomial $x^{7}-3 x^{5}-2 x^{3}-2 x^{2}+4 x-2$. The invariant trace fields were found with the help of the program snap ([9]).

\section{INEQUALITIES FOR THE EULER CLASS}

4.1. Thurston norm. We have seen from $\S 2$ and $\S 3$ that

$$
\left|e_{\sigma}(S)\right|<-\chi(S)
$$

for $S$ a fiber of $M$, and

$$
e_{\sigma}(S)=\chi(S) \bmod 2
$$

for any incompressible surface $S$, whenever $\sigma: K \rightarrow \mathbb{R}$ is a real place.

In [18], Thurston introduced a norm on $H_{2}(M, \partial M ; \mathbb{R})$ for $M$ irreducible and atoroidal. For a homology class $[S]$, the norm satisfies

$$
\|[S]\|=\inf _{S}-\chi(S)
$$

where the infimum is taken over all (possibly disconnected) representatives $S$ of $[S]$ with no spherical components.

A generalization of this norm, due to Gromov, measures a similar complexity amongst all immersed surfaces with no spherical components representing a given homology class. A theorem of Gabai ([6]) shows that these two norms are equal (after a suitable normalization); i.e. any immersed surface may be replaced by an embedded surface of no larger norm.

The key properties of the norm $\|\cdot\|$ are that the unit ball $\mathcal{P}(M)$ is a finite sided polyhedron, whose vertices are rational, and that there are a finite (possibly empty) collection of top dimensional faces $Q_{i}$ with the property that the integral homology 
classes $[S]$ representing fibrations of $M$ over $S^{1}$ are exactly those whose (positive) projective rays intersect the interiors of the $Q_{i}$. Such $Q_{i}$ are called fibered faces of $\mathcal{P}(M)$.

Our estimate implies the following:

Theorem 4.1. Let $M$ be a cusped hyperbolic 3-manifold, and suppose $\sigma: K \rightarrow \mathbb{R}$ is a real place with associated relative Euler class $e_{\sigma}$. Then for every fibered face of $\mathcal{P}(M)$ there is a vertex $V_{i}$ such that $e_{\sigma}\left(V_{i}\right) \neq\left\|V_{i}\right\|$. Similarly, there is a vertex $V_{j}$ such that $e_{\sigma}\left(V_{j}\right) \neq-\left\|V_{j}\right\|$.

4.2. Pseudo-rational surfaces. In [12], Long and Reid define a pseudomodular surface to be one whose cusp set is contained in $Q$.

We alter their definition slightly to adapt it to our context:

Definition 4.2. A subgroup $\Gamma<\operatorname{PSL}(2, \mathbb{R})$ is pseudo-rational if the traces of all elements are contained in $\mathbb{Q}$.

A discrete finite covolume pseudo-rational subgroup acts on $\mathrm{H}^{2}$ with quotient a pseudo-rational surface $S$. Such a surface in a hyperbolic 3-manifold is necessarily totally geodesic. Note that for us, pseudo-rational surfaces are always orientable.

Example 4.3. A thrice-punctured sphere is a (pseudo)-rational surface.

If $K$ is the trace field of $M$ and $\sigma: K \rightarrow \mathbb{R}$ is a real place, the traces of a pseudorational subsurface do not change. It follows that

$$
e_{\sigma}([S])= \pm \chi(S)
$$

for any pseudo-rational surface, and any real place $\sigma$. Consequently, we have the following corollary:

Theorem 4.4. Let $M$ be a cusped hyperbolic 3-manifold, and suppose $S \subset M$ is a pseudorational surface (possibly immersed). If $S$ is not (Gromov or Thurston) norm minimizing in its homology class, K has no real places.

In particular, if $M$ contains a separating pseudo-rational surface, its trace field has no real places.

Example 4.5. One method of constructing (pseudo)-rational surfaces is by covering thrice punctured spheres. A thrice-punctured sphere is always homologically essential, and therefore so is its preimage in a finite cover. But if such a finite cover has suitable symmetries, one might be able to find a (low genus) surface with a 2fold orientation-reversing fixed-point free symmetry, in the same homology class as the pseudo-rational surface. One can then cut along such a surface, and reglue the resulting boundary components to themselves to get a new manifold with the same trace field as the old, in which the pseudo-rational surface is homologically trivial.

A nice application of Theorem 4.4 is the following Corollary, which was suggested by A. Reid:

Corollary 4.6. Let $M$ be a fibered knot complement in a rational homology sphere whose trace field $K$ has odd prime degree. Then $M$ does not contain an immersed totally geodesic surface. 
Proof. Since $K$ has prime degree, it has no proper subfields other than $\mathbb{Q}$; in particular, any immersed totally geodesic surface $S$ has rational traces. Since $M$ is a knot complement in a rational homology sphere, its rational second homology is 1 dimensional. Since it is fibered, the rational homology is generated by the fiber $F$. It follows that $[S]=n[F]$ in homology for some nonzero integer $n$. Since $F$ is a fiber of a fibration, it is Thurston (and Gromov) norm-minimizing, and therefore

$$
-\chi(S) \geq-|n| \chi(F)
$$

Let $\sigma: K \rightarrow \mathbb{R}$ be a real place, and let $e_{\sigma}$ be the associated relative Euler class. Then we have

$$
\left|e_{\sigma}(S)\right|=|n| \cdot\left|e_{\sigma}(F)\right|<-|n| \chi(F) \leq-\chi(S)
$$

contrary to Theorem 4.4 .

For example, the knot $8_{20}$ in [17] (the complement is $\mathrm{m} 222$ in the census) is fibered, and has trace field generated by a root of $x^{5}-x^{4}+x^{3}+2 x^{2}-2 x+1$ which has degree 5.

4.3. Realizing Euler classes. If $\Sigma$ is a closed, orientable surface of genus $g \geq 2$, Goldman [8] showed that the $\operatorname{PSL}(2, \mathbb{R})$ representation variety of $\pi_{1}(\Sigma)$ has $4 g-3$ components, indexed by values of the Euler class on $\Sigma$ satisfying

$$
|e([\Sigma])| \leq-\chi(\Sigma)
$$

The Milnor-Wood inequality (c.f. [15], [21]) says that one cannot do better, even amongst $\mathrm{Homeo}^{+}\left(S^{1}\right)$ representations:

Theorem 4.7 (Milnor-Wood). Let $\Sigma$ be a closed surface of genus at least 1 , and let $\rho: \pi_{1}(\Sigma) \rightarrow$ Homeo $^{+}\left(S^{1}\right)$ be a representation with Euler class $e_{\rho}$. Then

$$
\left|e_{\rho}([\Sigma])\right| \leq-\chi(\Sigma)
$$

Similar theorems hold for surfaces with boundary, where one considers relative Euler classes.

For representations $\rho_{\sigma}$ coming from real places $\sigma$ of trace fields $K$, we have the additional constraint that $e_{\sigma}([\Sigma])=\chi(\Sigma) \bmod 2$. Modulo this constraint, we will see how to construct simple examples which realize every possible compatible combination of Euler characteristic and Euler class.

Definition 4.8. Let $M$ be a manifold, and $\mathcal{P}(M)$ the unit ball of the Thurston norm. A big diamond is a symmetrical 4-gon $D \subset \mathcal{P}(M)$ which is the intersection of $\mathcal{P}(M)$ with a two-dimensional plane $\pi$, and whose vertices are integer lattice points which generate the lattice of integral points in $\pi$.

Since the norm of every integer lattice point is at least 1, a "big diamond" is as big as possible, hence the name. Notice too that only cusped manifolds can have big diamonds in $\mathcal{P}(M)$.

Theorem 4.9. Let $M$ be a cusped hyperbolic 3-manifold. Suppose the trace field $K$ has a real place $\sigma$ with associated relative Euler class $e_{\sigma}$, and suppose further that the unit ball in the Thurston norm $\mathcal{P}(M)$ contains a big diamond $D$. Then for every integer $n<0$ and every integer $m$ with $|m| \leq-n$ and $n=m$ mod 2 there is an immersed incompressible connected surface $S_{n, m}$ in $M$ satisfying

$$
\chi\left(S_{n, m}\right)=n, e_{\sigma}\left(\left[S_{n, m}\right]\right)=m
$$


Proof. Let $V_{1}, V_{2}$ be surfaces representing the vertices of the big diamond $D$. Then $\chi\left(V_{1}\right)=\chi\left(V_{2}\right)=-1$ and therefore $\left|e_{\sigma}\left(V_{1}\right)\right|=\left|e_{\sigma}\left(V_{2}\right)\right|=1$. After replacing $V_{1}$ and/or $V_{2}$ with their negatives if necessary, we can assume

$$
e_{\sigma}\left(V_{1}\right)=1, e_{\sigma}\left(V_{2}\right)=-1
$$

For $p, q \geq 1$ let $V_{p, q}$ denote the Thurston norm-minimizing surface representing the homology class $p\left[V_{1}\right]+q\left[V_{2}\right]$. Since $D$ is a diamond, we have

$$
\chi\left(V_{p, q}\right)=-p-q
$$

Since $e_{\sigma}$ is linear, we have

$$
e_{\sigma}\left(V_{p, q}\right)=p-q
$$

If $p, q$ are coprime, then $V_{p, q}$ is represented by a connected surface. Otherwise, we have $p=a p^{\prime}, q=a q^{\prime}$ for some $a>1$ where $p^{\prime}, q^{\prime}$ are coprime. Then $\pi_{1}\left(V_{p^{\prime}, q^{\prime}}\right)$ has a subgroup of index $a$ which gives a connected incompressible immersed surface in $M$ with Euler characteristic $-p-q$ and Euler class $p-q$. Together with finite index subgroups of $\pi_{1}\left(V_{1}\right), \pi_{1}\left(V_{2}\right)$, this shows that every possibility is realized.

Example 4.10. The link 86 in Rolfsen's tables [17] has a complement whose unit ball in the Thurston norm is a big diamond, and has trace field generated by a root of $x^{3}-x^{2}+3 x-2$, which has a real place because the degree is odd (thanks to $\mathrm{N}$. Dunfield for finding this example.)

\section{REFERENCES}

[1] J. Brock, R. Canary and Y. Minsky, The classification of Kleinian surface groups, II: The Ending Lamination Conjecture, eprint, math.GT/0412006

[2] J. Button, Invariant trace fields of once-punctured torus bundles, Kodai Math. J. 28 (2005), no. 1, 181-190

[3] D. Calegari, Circular groups, planar groups and the Euler class, Geom. Topol. Monog. 7 (Proceedings of the Casson Fest) (2004), 431-491

[4] D. Cooper and D. Long, Remarks on the A-polynomial of a knot, J. Knot Theory Ramifications 5 (1996), no. 5, 609-628

[5] M. Culler, Lifting representations to covering groups, Adv. in Math. 59 (1986), no. 1, 64-70

[6] D. Gabai, Foliations and the topology of 3-manifolds, J. Diff. Geom. 18 (1983), no. 3, 445-503

[7] E. Ghys, Groupes d'homéomorphismes du cercle et cohomologie bornée, The Lefschetz centennial conference, Part III (Mexico City, 1984), 81-106 Contemp. Math., 58, III Amer. Math. Soc., Providence, RI, 1987

[8] W. Goldman, Topological components of spaces of representations, Invent. Math. 93 (1988), no. 3, 557607

[9] O. Goodman, Snap, available at http: //www.ms . unimelb.edu.au/ snap/

[10] A. Hatcher, A proof of a Smale conjecture, $\operatorname{Diff}\left(S^{3}\right) \simeq \mathrm{O}(4)$, Ann. of Math. (2) 117 (1983), no. 3, 553-607

[11] S. Jekel, A simplicial formula and bound for the Euler class, Israel J. Math. 66 (1989), no. 1-3, 247-259

[12] D. Long and A. Reid, Pseudomodular surfaces, J. Reine Angew. Math. 552 (2002), 77-100

[13] C. Maclachlan and A. Reid, The arithmetic of hyperbolic 3-manifolds, Graduate Texts in Mathematics 219 Springer-Verlag, New York, 2003

[14] K. Matsuzaki and M. Taniguchi, Hyperbolic manifolds and Kleinian groups, Oxford Mathematical Monographs, Oxford Science Publications The Clarendon Press, Oxford University Press, New York, 1998

[15] J. Milnor, On the existence of a connection with curvature zero, Comment. Math. Helv. 32 (1958), 215223

[16] W. Neumann and A. Reid, Arithmetic of hyperbolic manifolds, Topology '90 (Columbus, OH, 1990), 273-310, Ohio State Univ. Math. Res. Inst. Publ., 1, de Gruyter, Berlin, 1992.

[17] D. Rolfsen, Knots and Links, Corrected reprint of the 1976 original Mathematics Lecture Series, 7. Publish or Perish, Inc., Houston, TX, 1990 
[18] W. Thurston, A norm for the homology of 3-manifolds, Mem. Amer. Math. Soc. 59 (1986), no. 339, i-vi and $99-130$

[19] W. Thurston, Three-manifolds, foliations and circles II, privately circulated preprint, (1997)

[20] J. Weeks, SnapPea, available at http: / /www . geometrygames . org/SnapPea/

[21] J. Wood, Bundles with totally disconnected structure group, Comment. Math. Helv. 46 (1971), 257-273

Department of Mathematics, CAlifornia Institute of TeChNOlogy, PAsAdena CA, 91125 